\title{
Towards a shared method to classify contaminated territories in the case of an accidental nuclear event: the PRIME project
}

\author{
C. Mercat-Rommens ${ }^{1}$, S. Chakhar ${ }^{3,4}$, C. Barde ${ }^{1,2}$, \\ S. Roussel-Debet ${ }^{1} \&$ V. Mousseau ${ }^{3}$ \\ ${ }^{1}$ IRSN, Institute for Radioprotection and Nuclear Safety, France \\ ${ }^{2}$ University of Grenoble-UMR PACTE, France \\ ${ }^{3}$ University of Paris-Dauphine-LAMSADE, France \\ ${ }^{4}$ Magelis, France
}

\begin{abstract}
The analysis of the management of the accidentally radioactive contaminated areas such as those around Chernobyl nuclear power plant highlights the fact that the current spatial classification methods hardly help in recovering proper use of the contaminated territory. The cause is mainly to be searched for in the traditional construction of risks assessment methods; these methods rest on criteria defined by institutional experts, which are not applicable in practise because they are not shared by all the stakeholders involved in the management of the contaminated territories. Opposite such top-down tentative management, local efforts supported by Non-Governmental Organizations to restore life in the contaminated area seem to be more fruitful but very time and resources consuming and limited to the specific areas where they are experimented.

The aim of the PRIME project, in progress at the French Institute for Radioprotection and Nuclear Safety, is to mix the advantages of both approaches in building a multicriteria decision tool based on the territorial specificities. The criteria of the method are chosen and weighted with representatives of the territory's stakeholders (decision makers, local actors and experts) to warrant that all the points of view are taken into account and to enable the risk managers to choose the appropriate strategy in case of an accident involving radioactive substances. The area chosen for the pilot study is a $50 \mathrm{~km}$ radius territory around the nuclear sites of Tricastin-Pierrelatte in the lower valley of Rhône (France). One of the exploration questions of the PRIME project is whether a multicriteria method may be an appropriate tool to treat the data and make them visible and accessible for all the stakeholders.
\end{abstract}

Keywords: multicriteria analysis, radionuclide, post-accidental management. 


\section{Introduction}

The risk related to radiological and nuclear activities in France concerns 48 nuclear power reactors located on 19 geographic sites, 10 fuel cycle installations located on 6 sites, 61 research centres located on 4 sites, 10 installations which are being dismantled on 7 sites, 2 waste storage sites, industrial irradiators, and gamma radiography inspection equipment. In addition, this risk concerns 50000 local medical and research units located throughout France as well as transportations of radioactive material by road (300 000 transportations in 2004). The scope of French nuclear and radiological activities shows that the incorporation of territory characteristics is a potentially important component in the management of risks and especially accidental risk management.

The nuclear activities are effectively carried out to prevent accidents, but as it is not possible to guarantee zero risk, it is also necessary to limit the consequences of potential accidents. Two intervention phases are generally differentiated in case of an accident involving radioactive substances: an emergency phase involving a quick and organized response within the framework of intervention plans and a deferred phase, post-accident, which must be implemented in the medium or even long term in order to return to a situation considered acceptable by the various stakeholders involved. Even if the post-accident phase has to be implemented in continuity with the previous phase, the PRIME project is mainly focused on the development of methodological tools useful in this second phase.

The objective of the PRIME project is to develop, conjointly with the experts, the decision makers and representatives of the territory, a multi-criteria analysis method to characterize the contaminated territory that will be useful for the managers of the risk related to industrial accidents involving radioactive substances. The method is based on the ranking of the radio-ecological sensitivity factors of a territory with regard to radioactive pollution.

Is a $50 \mathrm{~km}$ radius territory around a nuclear site only sensitive to accidental nuclear pollution depending on its distance from the source, or are there criteria varying its sensitivity according to the nature and use of the land? What criteria are important for the people living in the territory and how are the criteria weighted against each other? What criteria might be used for decision-making? Would a multi-criteria method be a good tool to publish and make this data visible and accessible?

\section{Positioning and objectives}

The successive works carried out in France on post-accident situation management show that it is mandatory that this management is based on an anticipated characterization of the radiological status of the environment discussed globally and according to a strategy taking into account the inhabitants and their living conditions. The objective is to provide the managers and the civil 
society with a simple tool enabling a methodical choice of actions to undertake and the comprehension of such actions by all when health, economic, ecological and even social criteria are simultaneously involved.

The findings on the radiological consequences of radioactive releases, especially in the case of experience feedback from the Chernobyl accident, show that the consequences for man and the environment of such pollution depends on the importance and the nature of the pollution as well as on the polluted territory and its human and environmental context. This is also true for any industrial pollution. These consequences will be penalizing to different levels, whether expressed in economic, territorial image, toxicity or health risk terms, depending on the characteristics of the affected medium (environmental parameters) and according to its use (anthropologic parameters). The different media: urban, agriculture, forest, rivers, lakes or oceans show different sensitivities with regard to pollution. Within these major environmental components themselves, different natural or anthropologic factors, specific to the ecosystem involved, determine the response of the environment to pollution at a given moment. In an agricultural area, for example, the type of culture and the vegetative cycle time constitute the major sensitivity factors. Wheat and dairy products coming from a surface affected by the same pollution will also have very different respective levels of contamination. The remnants from this contamination in successive cultures will greatly depend on the soil characteristics. In addition, generally speaking, all characteristics intrinsic to an ecosystem and affecting transfer of pollutants invest a territory with a specific sensitivity with regard to pollution. The same statement applies to anthropologic factors such as cultural practices, use of fertilizers, irrigation, sowing periods or animal production (animal feeding, their presence outside). All these factors are later on called the sensitivity factors (meaning radiological sensitivity). The radiological sensitivity of a territory is therefore defined by two components: environmental and anthropologic. The relative weighting of these components may change over time.

If a territory is sensitive to pollution native to this territory, it is currently difficult to compare the global sensitivity of different territories: is it more serious to have a major stock of pollutants in a natural space with little human presence or to have a low concentration of the same pollutants in a watercourse used extensively for irrigation? The radiological sensitivity is a concept making it possible to represent the intensity of the response of a territory to pollution. The perspectives of use of this concept have been explored at the IRSN since 2003 as part of the SENSIB project supported by IRSN and ADEME (Mercat-Rommens et al [1]). The concepts studied in SENSIB show interesting perspectives for application in the different stages in the life of a nuclear installation (chronic discharge, accidental context, dismantling). The objective of the PRIME project is to develop an application of the radiological sensitivity concept at the scale of a territory in the post-accidental context.

The PRIME project is designed as a partnership between scientific laboratories (IRSN and other risk expert institutes, Universities: ParisDauphine/LAMSADE, Grenoble/UMR PACTE, etc.), representatives for the 
public authorities (ASN/Division of Lyon, Prefecture, town hall) and also representatives for the civil society (Local Information Commissions). Therefore, this project is a "complex" project according to Le Cardinal et al [2], being "a system in which different players build representations; use rationalities; undergo constraints; are based on assessment criterion and elaborate their own objectives". That also makes PRIME a project to explore the forms of innovative cooperation usable for industrial risks management. In this challenge, the PRIME project benefits from the experience acquired by the IRSN from opening of the scientific expertise to stakeholders involved, especially as part of the framework of the Nord-Cotentin Radiological Group works (MercatRommens and Sugier [3]).

This project must in the end provide territory characterization usable within the framework of risk management and evaluation for man and for the environment.

The multi-criteria analysis is used here in its original manner as it is used to explain the environmental assessment criteria (sensitivity indicator) supporting partnership decisions with the stakeholders involved. The exhaustive list of sensitivity factors proposed by the stakeholders involved and their systematic examination should eliminate some limits of these methods observed in the past, when the factors were listed unilaterally by the experts and quickly questioned by the other players.

\section{Methodology and case study}

The territory notion selected for the PRIME project is the notion of a territory affected by the accident according to the meaning of "risk territory". Therefore, the territory involved is not necessarily an administrative unit or a territory in which human activities are homogeneous. The management of the border differences between the "risk territory", the "administrative territory" and the "social territory" is one of the challenges of the PRIME project as the availability of data does not necessarily cover the same scales as the territories and the decision coverage territory will probably not be the same. The studied territory of the PRIME project is located in the Southeast of France, in the Basse Vallée du Rhône (figure 1). The accidental pollution source will be a virtual point located on the nuclear site of Tricastin. However, the project also covers accidental nuclear pollution that could occur from the Cruas and Marcoule nuclear sites.

In terms of the affected territory, the study territory is located globally within a fifty kilometres area around the three nuclear sites and the territory studied covers also the Rhône river downstream from Marcoule and the nearby coastal territories.

The media treated are the atmosphere, the soil, the agricultural productions, the river domain, the water supplies and the marine coastal territories. Interfaces between these media are taken into account. The different ensuing anthropologic actions are also taken into account: foodstuff transformation, sales chains related to agricultural products from the contaminated territory, water uses. 


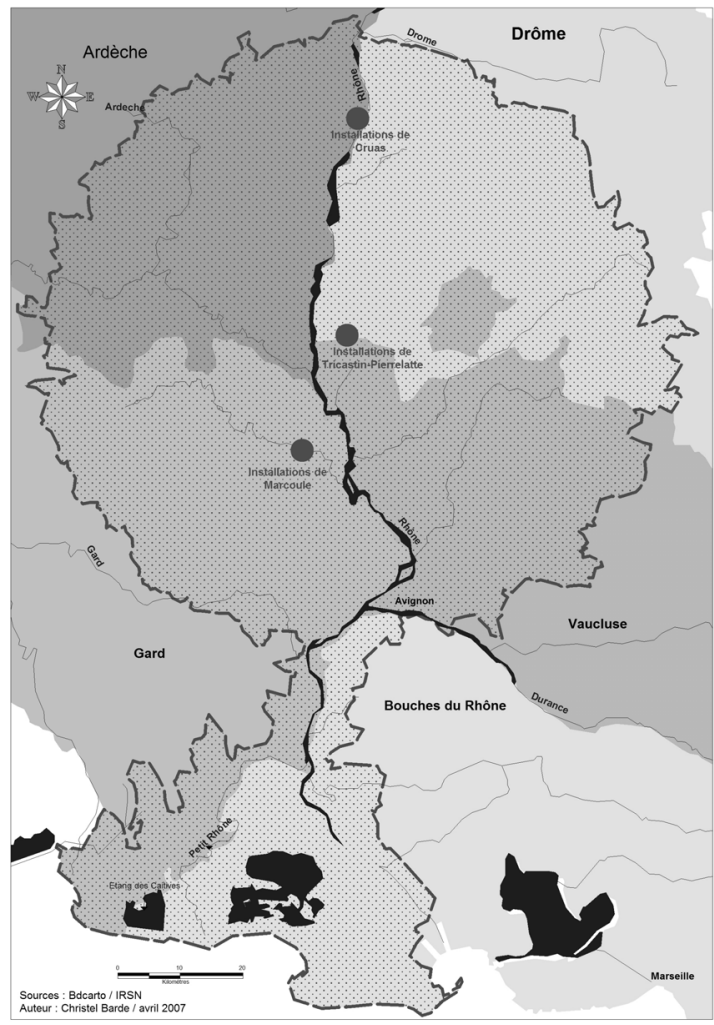

Figure 1: $\quad$ Study area.

Performance of the PRIME project steps has required federation of available radio-ecological data (field data, modelling, experimental results), as well as territory data and then the processing of these with the common approach defined by the project. The assessment method of the radiological sensitivity indicators invoked classic impact calculation models for radionuclides used at the IRSN: CASTEAUR code for river discharges (Duchesne et al [4]), ASTRAL code for forest ecosystem and food chain contamination following accidental radioactive pollution (Renaud et al [5], Calmon and Mourlon [6]), integrating the spatial variability of parameters.

Assessment of the radiological sensitivity unavoidably leads to the calculation of different sensitivity indicators. In the radio-ecological domain this concerns, for example, the volumetric activities in the water table, the surface activities on soils intended for different uses, the total activities "produced" on agricultural lands, the radionuclide flows in running streams. In addition, territory sensitivity indicators of different natures complete the radiological sensitivity indicators: demographic indicators (temporary or resident population, urbanization, etc.), "ecological" indicators (presence of protected areas or species), socio-economic 
indicators (value of agricultural production, storage/distribution costs, countermeasure costs, indirect costs for abandonment of tourist territories, degradation of the territory image...).

The PRIME project is studying the methods available to rank the radiological sensitivity of a territory and to find a means of comparing them one against the other. The data processing methods explored in the PRIME project are those of the multi-criteria analysis (AHP, MAUT, ELECTRE, PROMETHE...) (Keeney and Raiffa [7], Maystre et al [8]).

Recourse to a multi-criteria analysis method makes it possible in particular to model and formalize decision preparation. In this line of thought, recourse to multi-criteria methods increases the chance of improving the transparency of the decision process and the traceability of this process. This time, the opinion of involved stakeholders is strongly requested in particular to guarantee the acceptability of future use of the tool.

Three main steps are consecutive in the development of a multi-criteria analysis method, being:

- The development of the assessment table for which the radiological sensitivity of the components of a territory are assessed using specific sensitivity indicators of each medium. Each sensitivity indicator may be weighted with the approval of the stakeholders involved.

- The performance of the aggregation procedure which makes it possible to obtain the global preferences from the over-classing relations, deduced from the assessment table. During this step, it is necessary to choose the type of multi-criteria analysis method (sort method, classification method, etc.). This step introduces the discordance or concordance threshold notion. The setting of a single concordance threshold makes it possible to determine the value under which the over-classing hypothesis of an action in relation to another action is to be rejected. The discordance threshold makes it possible to determine the maximum tolerance so that an over-classification hypothesis will not be rejected. These thresholds also make it possible to discuss what opposition and compromise levels are acceptable between the different players and may make it possible to take diverging positions between players into account.

- The ruggedness analysis of the result, which then makes it possible to test whether the results are not modified significantly when the parameters vary around their initial value and therefore to know whether the recommendation is rugged. The parameters that may be varied are the range of radiological sensitivity indicators values (or the notation if they are quality criteria), the indicator weight, the discordance and/or concordance threshold. An in-depth analysis of the ruggedness will in particular make it possible to compensate for the subjective nature of certain parameters.

At the present time, the PRIME project had partly performed the first of the three steps and prepared the tools necessary to perform the two following steps. 


\section{Results}

The restitution of PRIME project results can take different formats: assessment guide for the radiological sensitivity, mapping of iso-sensitivity territories and sensitivity scale (Figure 2). The format of the scale was discussed with partners and especially with the representatives of decision-makers: number of levels, threshold values, choice of aggregation in a single index (like in the international INES scale for damage related to nuclear accidents), or aggregation around several indexes (like in the French BARPI scale for damage related to industrial accidents). The scale obtained will be coupled with a tool making it possible to make a cross reference between a given situation (map of radionuclide deposition in each town) and a scale level.

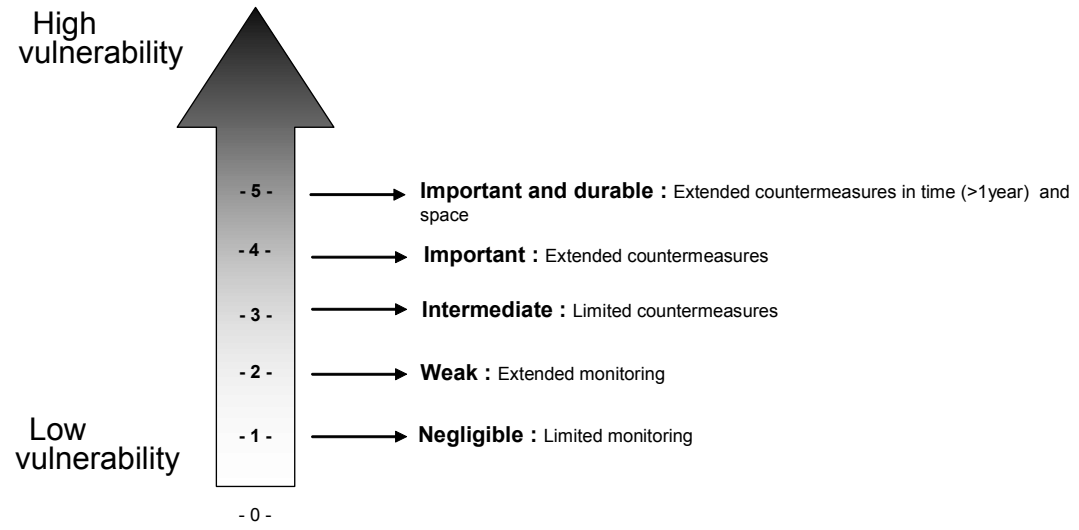

Figure 2: $\quad$ The PRIME proposition of classification scale.

\section{Conclusion}

PRIME is an ongoing participative research that tries to build, in a concerted manner, a spatial classification tool to support efficient post-accidental strategies. Two main objectives are challenged in PRIME: a social challenge to identify with all the stakeholders the best criteria to characterize the state of the environment and a technical challenge to make the criteria accessible for the citizens and operational for the decision-making via a multicriteria method coupled with a GIS.

\section{References}

[1] Mercat-Rommens C., Roussel-Debet S., Briand B., Durand V., Besson B., Renaud P., The radioecological sensitivity of territories: towards an operational tool through the SENSIB Project, Radioprotection, 42, pp. 277295, 2007. 
[2] Le Cardinal G., Guyonnet J.F., Pouzoullic B., La dynamique de la confiance : construire la coopération dans les projets complexes. Dunod: Paris, pp. 246, 1997.

[3] Mercat-Rommens C.,Sugier A., Le Groupe Radioécologie Nord-Cotentin, une expérience scientifique d'expertise pluraliste. Proc. of the conference "Les nouveaux outils pour décider ensemble», Eds. Université ParisDauphine: Paris, 2006.

[4] Duchesne S., Boyer P., Beaugelin-Seiller K., Sensitivity and uncertainty analysis of a model computing radionuclides transfers in fluvial ecosystems (CASTEAUR): application to ${ }^{137} \mathrm{Cs}$ accumulation in chubs. Ecological Modelling, 166(3), pp. 257-276, 2003.

[5] Renaud P., Stapel R., Maubert H., Bleher M., Wirth E., Comparative study of the PARK and ASTRAL post-accidental decision support software. Health Physics, 76(5), pp. 502-509, 1999.

[6] Calmon P., Mourlon C., ASTRAL V2.2, A new version to better assess post-accidental situations. Radioprotection, 40(Sup. 1), pp. S839-S844, 2005.

[7] Keeney G.A., Raiffa H., Decisions with multiple objectives: preferences and value trade-offs. Wiley: New-York, 1976.

[8] Maystre L.Y., Pictet J., Simos J., Méthodes multicritères ELECTRE, Presses Polytechniques et Universitaires Romandes. Collection Gérer l'environnement, pp. 1-323, 1994. 